\title{
Research on of Highline Cable of Alongside Replenishment at Sea
}

\author{
Yunpeng ZHAO \& Nan LI \\ Service College, Naval Engineering University, Tianjin 30045C.T.Zhao
}

\begin{abstract}
In view of the present situation of that has not had an overhead cable model can be used in the process of developing equipment of replenishment at sea, the multibody dynamics theory was introduced into the Highline modeling. Establish method of multi-rigid-body dynamics model, rigid-flexible-mixed-body dynamics model and the multi-flexible-body dynamics model of the rope was researched, and the calculation formulas of three kinds of multi body dynamics model of the rope were deduced. Combining with the actual situation, the concluded has been gain which are as follows: (1) The multi-body dynamics model of rope can simulate the Highline replenishment at sea; (2) multi-flexible-body dynamics model of the rope is more close to the actual structure of overhead cable than the other two multi-body dynamics models of the rope, the dynamic calculation results of which is closer to the actual situation, and it has certain use value in equipment development of the replenishment at sea .

KEYWORD: Alongside Replenishment at Sea; Highline Cable; Multibody System Dynamics; Multi-body System; Multi-rigid-body System; Mixed Rigid Flexible Body System
\end{abstract}

Highline is the bridge between the ship and the supply ship as an important part of replenishment equipment, Which charges with carrying effect or directly is a transmission channel. Therefore, it is the first to solve that establish of reasonable and accurate Highline dynamics model in the sealift equipment developed in the process of the problem. At present, the study on the model of highline replenishment at sea mainly focus on statics highline cable model based on the theory of mechanics of rigid body [1]. This study only stay in the static analysis of the twodimensional plane, which divides the cable into a number of mass points, find position and force condition of the points set by the vibration mechanics calculation, then each quality points are connected together by a curve fitting method. This method has too more assumptions, and the Highline was not considered as mass distribution of entities, the results have been unable to completely close to the actual situation, also cannot be directly applied in engineering practice.

This paper study of the dynamic modeling method of maritime replenishment rigs by using of multibody dynamics theory, and put forward that the Highline is as a multi-flexible-body system, a rigidflexible-body system and a multi-rigid-body system. Then it establish the dynamics of multi-flexible-body system model, rigid-flexible-body system and multirigid-body system of maritime replenishment rigs.

Flexible multi-body system is a system that the research object is composed of a plurality of flexible body. The dynamic analysis of the system must consider position, velocity, acceleration, angular velocity and angular acceleration of each rigid body unit, and the flexible connecting role between adjacent units and flexible body, and how to transfer the position, velocity, acceleration, angular velocity and angular acceleration. Highline belongs to a flexible slender body, which can be model by adopting the segment flexible body method of the multi-body dynamics theory of HUSTON[2-3]. Tthe rope is discretized into a number of segments of rope units. Each rope is look at a elastic cylindrical beam, between the rope section is composed of a flexible hinge connected to form a flexible multi body system.

\section{BUILDING THE PATH MATRIX T}

The topological relations of the multi-body system of rope is as shown below:

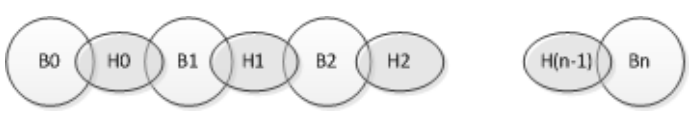

Fig. 1 rope topological relation graph 
According to the topological relationship and the definition of the path matrix, the path matrix of the rope multi-rigid-body system[4] is:

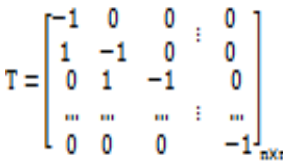

\section{THE KINEMATICS EQUATION OF FLEXIBLE JOINTS}

The linking joints is assumed to be flexible. The hinge joints of rigid body[5-6] are respectively $\mathrm{P}$ and $\mathrm{Q}$. The location of $\mathrm{P}$ in local coordinate system is ${ }^{\mathrm{e}_{\mathrm{Pi}}}$. The location of $\mathrm{Q}$ in local coordinate system is ${ }^{{ }}{ }_{\mathrm{Qi}}$. The force and moment of the joint are respectively $F$ and $\mathrm{T}$. The relative displacement, rotation, velocity and angular velocity of ${ }^{{ }^{{ }{ }_{i i}}}$ with respect to ${ }^{{ }^{{ }_{\mathrm{Pi}}}}$ are:

$\left[\begin{array}{l}F_{x} \\ F_{y} \\ F_{z} \\ T_{x} \\ T_{y} \\ T_{z}\end{array}\right]=\left[\begin{array}{cccccc}K_{11} & 0 & 0 & 0 & 0 & 0 \\ 0 & K_{22} & 0 & 0 & 0 & 0 \\ 0 & 0 & K_{33} & 0 & 0 & 0 \\ 0 & 0 & 0 & K_{44} & 0 & 0 \\ 0 & 0 & 0 & 0 & K_{55} & 0 \\ 0 & 0 & 0 & 0 & 0 & K_{66}\end{array}\right]\left[\begin{array}{l}R_{x} \\ R_{y} \\ R_{z} \\ \theta_{x} \\ \theta_{y} \\ \theta_{z}\end{array}\right]-\left[\begin{array}{cccccc}C_{11} & 0 & 0 & 0 & 0 & 0 \\ 0 & C_{22} & 0 & 0 & 0 & 0 \\ 0 & 0 & C_{33} & 0 & 0 & 0 \\ 0 & 0 & 0 & C_{44} & 0 & 0 \\ 0 & 0 & 0 & 0 & C_{55} & 0 \\ 0 & 0 & 0 & 0 & 0 & C_{66}\end{array}\right]\left[\begin{array}{c}v_{x} \\ v_{y} \\ v_{z} \\ \dot{\theta}_{x} \\ \dot{\theta}_{y} \\ \dot{\theta}_{z}\end{array}\right]+\left[\begin{array}{c}F_{x 0} \\ F_{y 0} \\ F_{z 0} \\ T_{x 0} \\ T_{y 0} \\ T_{z 0}\end{array}\right]$

Where $R 、 \theta 、 V 、 \dot{\theta}$ are the relative displacement, rotation, velocity and angular velocity respectively of

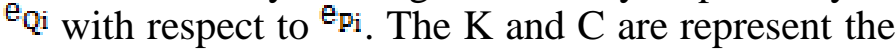
stifness and damping coefficient of the material respectively.

\section{THE ESTABLISHMENT OF THE ELEMENT COORDINATE SYSTEM}

Each unit of the Highline multi-flexible body dynamics model[7] is elastic cylindrical beam unit. Any particle of the unit moves in the space, and moves relative of the center of mass. So it must to build a body fixed coordinate system in unit centre position to calculate the relative position of inertial motion unit, motion and attitude of coordinate system, and build each particle floating coordinate system to calculate the unit itself deformation in the establishment of coordinate system.

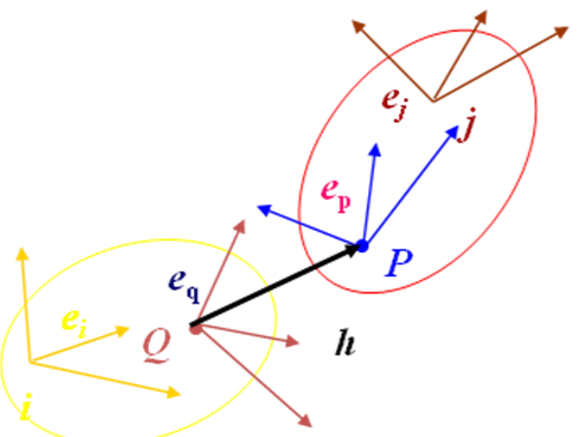

Fig. 2 adjacent rope unit coordinates $e_{q}, e_{p}$ are the body fixed coordinate system of Unit Q, P, ${ }_{i} 、 e_{j}$ are the floating coordinate system of particles $i$ and $j$ on the units $Q$ and $P$.

The particle $\mathrm{K}$ is unit relative to the inertial coordinate system for the radius vector:

$\rho^{k}=\rho_{0}^{k}+u^{k}=\rho_{0}^{k}+\Phi^{k} a$

In the formula, $\rho_{0}^{k}$ is the vector of unit centre relative to the geodetic coordinate system, ${ }^{\varphi}$ and $a$ are the translational modal vector array and modal coordinate of the particle $\mathrm{K}$ relative to unit on centre.

\section{THE ABSOLUTE ANGULAR VELOCITY OF ROPE UNIT}

Due to the difference between the three models, the absolute angular velocity formulas of three models have the difference.

Considered the rigid drive between the rope units of the multi-rigid-body models of rope, the angular velocity of the formula is as:

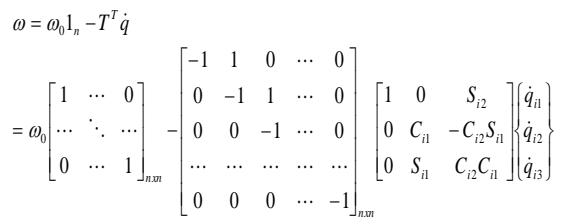

Added the damping at the connected between units of rigid- flexible-body hybrid model of rope, the angular velocity of the formula is as:

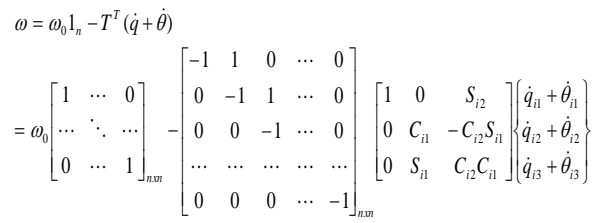

Based on the rigid-flexible -body hybrid model of rope, make the rope unit to flexible, the angular velocity of the formula is as:

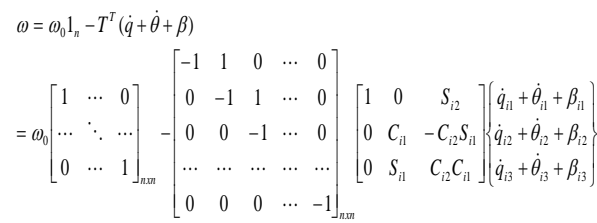

\section{ESTABLISHING A PATH VECTOR ARRAY}

The center of mass of any unit in the inertial coordinate system on position can be formed by the superposition of a series of vector[8].

Definition: the origin of inertial coordinate system is $\mathrm{O}, r_{0}$ is the radius vector from the inner hinge to the $\mathrm{O}$ point. $C_{k k}$ is the radius vector from the inner hinge of $B_{k}$ to the mass center of $B_{k} . C_{k i}$ is the radius 
vector from the outer hinge of ${ }^{B_{k}}$ to the mass center of $B_{k}$, So:

$\mathrm{d}_{\mathrm{ki}}= \begin{cases}0 & (\mathrm{i}<k) \\ -\mathrm{C}_{k k} & (\mathrm{i}=\mathrm{k}) \\ -\mathrm{C}_{k \mathrm{k}}+\mathrm{C}_{\mathrm{ki}} & (\mathrm{i}>\mathrm{k})\end{cases}$

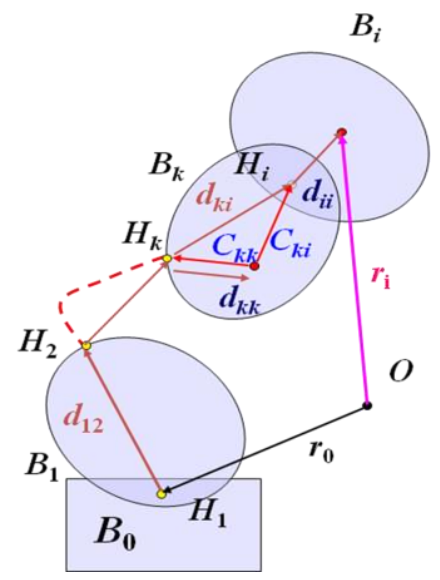

Fig. 3 overhead cable channel vector

It established the highline path vector array according to the channel vector formula:

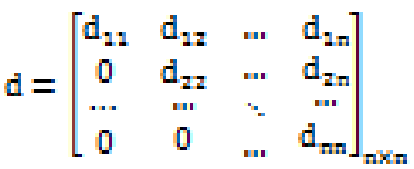

The position of mass center of any rope unit is: $r_{i}=r_{0}+\sum_{k=1}^{n} d_{k i}$.

The position of any mass point of rope unit is: $r_{i}=r_{0}+\sum_{k=1}^{n}\left(d_{k i}+R_{i}\right), \quad R_{i}$ is the radius vector from the point to the mass center.

\section{THE ABSOLUTE VELOCITY OF ROPE UNIT CENTER-OF-MASS}

When the rope unit is rigid, the velocity of rope unit $i$ $s$ the absolute velocity of center-of-mass of rope rigid unit. When the rope unit is a flexible body, the veloc ity of rope unit[9-10] is the sum of absolute velocity o f infinitesimal.

The absolute velocity of rope unit center-of-mass of multi-rigid-body system rope is:

$V=V_{0} 1_{n}-d^{T} \times \omega$

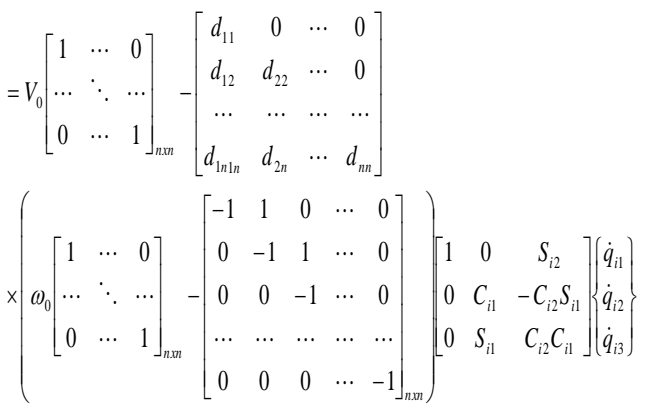

The absolute velocity of rope unit center-of-mass of rigid- flexible -body system rope is:

$$
\begin{aligned}
& V=V_{0} 1_{n}-d^{T} \times \omega+V \\
& =V_{0}\left[\begin{array}{ccc}
1 & \cdots & 0 \\
\cdots & \ddots & \cdots \\
0 & \cdots & 1
\end{array}\right]_{n n n}-\left[\begin{array}{cccc}
d_{11}+R_{11} & 0 & \cdots & 0 \\
d_{12}+R_{12} & d_{22}+R_{22} & \cdots & 0 \\
\cdots & \cdots & \cdots & \cdots \\
d_{1 n}+R_{1 n} & d_{2 n}+R_{2 n} & \cdots & d_{n n}+R_{n n}
\end{array}\right] \\
& \left.\times\left[\begin{array}{ccc}
1 & \cdots & 0 \\
\cdots & \ddots & \cdots \\
0 & \cdots & 1
\end{array}\right]_{n ! n}-\left[\begin{array}{ccccc}
-1 & 1 & 0 & \cdots & 0 \\
0 & -1 & 1 & \cdots & 0 \\
0 & 0 & -1 & \cdots & 0 \\
\cdots & \cdots & \cdots & \cdots & \cdots \\
0 & 0 & 0 & \cdots & -1
\end{array}\right]_{n n}\right\}\left[\begin{array}{ccc}
1 & 0 & S_{i 2} \\
0 & C_{i 1} & -C_{i 2} S_{i 1} \\
0 & S_{i 1} & C_{i 2} C_{i 1}
\end{array}\right]\left\{\begin{array}{c}
\dot{q}_{i 1}+\dot{\theta}_{i 1} \\
\dot{q}_{i 2}+\dot{\theta}_{i 2} \\
\dot{q}_{i 3}+\dot{\theta}_{i 3}
\end{array}\right\}
\end{aligned}
$$

The absolute velocity of rope unit center-of-mass of multi- flexible -body system rope is:

$$
\begin{aligned}
& V=V_{0} 1_{n}-d^{T} \times\left(\omega+V+\dot{\rho}+V_{r}^{K}\right. \\
& =V_{0}\left[\begin{array}{ccc}
1 & \cdots & 0 \\
\cdots & \ddots & \cdots \\
0 & \cdots & 1
\end{array}\right]_{n \cdot n}-\left[\begin{array}{cccc}
d_{11}+R_{11} & 0 & \cdots & 0 \\
d_{12}+R_{12} & d_{22}+R_{22} & \cdots & 0 \\
\cdots & \cdots & \cdots & \cdots \\
d_{1 n}+R_{1 n} & d_{2 n}+R_{2 n} & \cdots & d_{n n}+R_{n n}
\end{array}\right] \\
& \times\left[\left[\begin{array}{ccc}
1 & \cdots & 0 \\
\cdots & \ddots & \cdots \\
0 & \cdots & 1
\end{array}\right]_{n n n}\left[\begin{array}{ccccc}
-1 & 1 & 0 & \cdots & 0 \\
0 & -1 & 1 & \cdots & 0 \\
0 & 0 & -1 & \cdots & 0 \\
\cdots & \cdots & \cdots & \cdots & \cdots \\
0 & 0 & 0 & \cdots & -1
\end{array}\right]_{n n n}\right)\left[\begin{array}{ccc}
1 & 0 & S_{i 2} \\
0 & C_{i 1} & -C_{i 2} S_{i 1} \\
0 & S_{i 1} & C_{i 2} C_{i 1}
\end{array}\right]\left[\begin{array}{l}
\dot{q}_{i 1}+\dot{\theta}_{i 1}+\beta_{i 1} \\
\dot{q}_{i 2}+\dot{\theta}_{i 2}+\beta_{i 2} \\
\dot{q}_{i 3}+\dot{\theta}_{i 3}+\beta_{i 3}
\end{array}\right.
\end{aligned}
$$

\section{SUMMARY}

This paper has studied the dynamic model of multi-ri gid-body system, rigid-flexible-body system and mul ti-flexible-body of rope based on the multi-body dyn amics theory. The highline cable of replenishment at sea was analysed by applying the three kinds of dyna mic models. Combining with the actual conditions of highline cable, it is convinced that the multi-flexible -body system dynamic model of rope is reliable beca use of good agreement of simulation results and pract ice.

\section{REFERENCES}

[1] Ma Rengang, Zhang Liangxin. Static analysis cable of dry cargo replenishment system overhead. ship science and technology, 2012, (2):54-56.

[2] Huston R L, Passerello C E,Harlow M W.Dynamics of Multi-rigid-body System. ASME Journal of Applied Mechanics, 1978, 45(4): 889 894.

[3] Huston R L, Passerello C E. Multibody Structural Dynamics Including Translation Between the Bo-dies. Computers and Structures, 1980, 11: 713 720.

[4] HONG Jia-zhen. Computational dynamics of multi-body systems. Higher Education Press, Beijing, 1999.

[5] Yao Tingqiang, Wang Lihua Multi-bodies contact dynamics of ball bearing. Journal of aerospace power, 2013, (7): 45-48.

[6] Rui Xiaoting, Rong Bao. Progress research of translation matrix. mechanical multi-body system transfer, 2012, (1): 18-22. 
[7] Miu Jiancheng. calculation of flexible multi-body dynamics method and large research and analysis of antenna power. Shanghai Jiao Tong University, 2008:1722.

[8] Zhang Guoqing. modeling and control of flexible multibody system. University of Science \& Technology China, 2008: 45-51.
[9] Guo Tianeng. stay cable vibration control of space flexible structure. Tsinghua University, 2007:38-43.

[10] Ji Jun. deformation mechanism motion and flexible beam system analysis mechanics method [J]. Shanxi building 2011, (17): 23-26. 